\title{
Preface to the Inaugural Issue
}

\author{
Juan Carlos Augusto \\ Department of Computer Science, Middlesex University, London, UK \\ E-mail: j.augusto@mdx.ac.uk
}

\section{Introduction}

This Inaugural Issue of the Journal of Smart Cities and Society (SCS) starts a publication journey in one of the most recent and exciting developments at the crossroads of the scientific and technological worlds. Here we open a forum for the discussion and progress of the concept of Smart Cities and of its impact in society at large. SCS will focus on the already existing and well-known concept of Smart Cities [1] which amalgamates smart infrastructures with human's needs through services at a community level. Smart cities is an emerging concept which has been gaining momentum as the technology available becomes more sophisticated and provides continuous stimuli to solve some of the growing concerns due to demographical growth.

There are already various "smart..." areas of technology quite consolidated and making their way into business and society [see for example: [2,3]]. These smarter systems are supported by various areas in computer science, for example sensor networks and context-aware software, which facilitates delivering more specific services to humans in more specific daily life contexts and better aligned to their individual preferences. This has not been a sudden revolution, rather another stage in technological evolution. With areas such as artificial intelligence maturing and basic infrastructures such as high level connectivity always increasing efficiency. It seems quite a natural progression for society in general and for our technical communities as well, to move from the various current "smart ..." silos, such as smart homes, smart cars, smart transportation, smart food grow, smart health, to be interconnected through an overall area which focuses on the multiple combinations and on their cooperation to achieve benefits for society none of those single areas can achieve in isolation.

Hence there are strong motivations for their emergence and consolidation and on the other hand given the combination and complexity of resources required is a topic which will require a long and patient discussion. Design, experimentation, community involvement, cultural adaptations into different parts of the planet, and much more will be required to create services which work effectively in the real world.

Another interesting facet of this area is its essential multi-disciplinary nature. Technology acts in this area as a social bonding element as for each of the services which underpins, so many other groups of professionals are required in order to create meaningful services. It amalgamates a myriad of diverse areas such as computing, various branches of engineering, architecture, social sciences, environmental sciences, emergency services, city planners, and many more, ultimately citizens themselves as they know which services they need. Hence this area is a fascinating opportunity for society to grow in a more organic way with a more democratized and decentralized way of using progress for common good.

This Inaugural Issue contains seminal work from eight different teams which offer a complementary analysis of a number of important areas:

"Civic Technology: A Chutes and Ladders Analysis of Transformative Potential" by P. James covers the importance of citizen-centric view of Smart Cities as well as the potential threats to achieving this.

"The analysis of security and privacy risks in smart education environments" by A. Kamenskih analyzes the development of safer smart education as well as its technological sustainability. 
"Data Governance in Smart Cities: Challenges and Solution Directions" by S. Choenni, M. Bargh, T. Busker, and N. Netten identifies key functionalities for governance of a data ecosystem within smart cites as well as organizational aspects to sustain it.

"Sampling Blockchain-enabled Smart City Applications among South Korea, the United States and China" by S. Kim, R. Liao, A. Zhang, W. Zheng, Z. Hu and Z. Sun reports on a transnational assessment of blockchain centred data security issues based on different administration models.

Lastly, "A smart governance diffusion model for blockchain as an anti-corruption tool in Smart Cities" by C. Parenti, N. Noori, and M. Janssen provides insights for improving the understanding of contingencies for technology diffusion in Smart Cities and considers blockchain as a mitigator of vulnerabilities.

The foundational editorial team of this journal expects that the contributions of this Inaugural Issue will provide an initial agenda which inspires and guides other colleagues in this developing community.

We encourage all sectors of society to engage in this technical conversation as our view of this area as a multidisciplinary one which will require the input of various different professions and different levels of involvement within urban environments to produce effective innovation.

\section{References}

[1] J.C. Augusto, Smart cities: State of the art and future challenges, in: Handbook of Smart Cities, Springer, Cham, 2020. doi:10.1007/978-3030-15145-4.

[2] J.C. Augusto, M. Huch, A. Kameas, J. Maitland, P. McCullagh and J. Roberts, Handbook of Ambient Assisted Living - Technology for Healthcare, Rehabilitation and Well-Being, AISE Series, Vol. 11, IOS Press, 2012.

[3] H. Nakashima, H. Aghajan and J.C. Augusto, Handbook of Ambient Intelligence and Smart Environments, Springer, 2009. 